\title{
Twin Policy Management Model for Energy Harvesting Wireless Sensor Networks
}

\author{
Anuj Kumar Jain \\ Ph. D. Scholar \\ Comp. Sc. \& Engg. \\ Department \\ Maharishi Markandeshwar \\ University Mullana, Haryana, \\ India
}

\author{
Sandip Goyal, PhD \\ Professor \& Head \\ Comp. Sc. \& Engg. \\ Department \\ Maharishi Markandeshwar \\ University Mullana, Haryana, \\ India
}

\author{
Devendra Prasad, PhD \\ Professor \\ Comp. Sc. \& Engg. \\ Department \\ Chandigarh Group of Colleges, \\ Landran, Mohali, Punjab, \\ India
}

\begin{abstract}
This paper proposes a twin policy model to manage energy for a prolonged network lifetime of a sensor network. Based on the total remaining energy and Probability index calculation to harvest energy in the next round, and an expected energy achieving constant it decides which policy is to be implemented. It is also proposed that a switch between continuous model and partially event triggered model of data reporting. First Policy makes the network to work on continuous model and second policy governs the network for event triggered model. This paper uses a heterogeneous model where a small fraction of nodes have harvesting potential to harvest ambient energy. A multi-hop data forwarding model is used for the communication of data to the sink and to forward command to the nodes.
\end{abstract}

\section{General Terms}

Wireless Sensor Networks, Energy harvesting, Heterogeneous network, distributed clustering.

\section{Keywords}

Energy harvesting, Event triggered model, Continuous data reporting model.

\section{INTRODUCTION}

Wireless Sensor Networks (WSNs) are actually a collection of small nodes which are equipped with capabilities of sensing, computation, communication and sometimes movement. The nodes collaboratively work to sense either a physical phenomenon or environmental phenomenon. The sensor nodes also have constraints of energy due to limited battery power, bandwidth, small memory storage, limited computation capability and small transmission ranges. For the hostile areas where the battery cannot be changed or charged manually, Power is a critical and a high priority constraint to tackle with. Thus, it becomes one of the key areas of research to provide a longer network lifetime in such hostile areas. After deploying sensor nodes in such areas it is expected from the network to provide services as long as possible.

To address the energy constraint one need to identify the key components that consume most of the energy of a sensor node. Transceiver of a sensor node is one such component. By optimizing the operations of transceiver one can minimize the consumption of energy. Less number of data/command packets exchanged between the sink and sensor node would mean less energy dissipation. Another approach to tackle energy constraint is to harvest energy from the ambient energy sources. Even a small amount of harvested energy can provide a support to crucial communication in hostile areas [10].

\section{RELATED WORK}

D. Niyato et. al. in 2007 proposed a strategy to save energy by using sleep and awake pattern for solar powered wireless sensor/mesh networks [8]. They modeled the energy harvesting scheme as Markov chain.

Winston K.G. Seah et.al. in 2009 proposed an approach to power WSNs by converting the ambient energy available from the environment into an electrical energy to provide power to the sensor nodes [1]. Problem with renewable energy technology(e.g., solar and wind) is that it cannot be available all the time continuously. It can also not be available at all the places. Therefore it is highly unreliable. The sensor node has to harvest and store whatever amount of energy they can.

Focus on self-sustaining sources of energy to power the wireless sensor nodes has been there for a while now. Voigt et al. reported in 2003 proposed a power-aware protocol [3], basically is a variant of Directed Diffusion. In this scheme gradients flow preferably through solar powered nodes whereas the network do not carry out the power management in reported sense.

Satyajayant Misra et.al. in 2011 presented the constrained relay node placement(CRNP) problem for an energy harvesting wireless sensor network in [4].This scheme is concerned with deploying relay nodes at candidate locations inside the network to address requirements of connectivity and network In an energy harvesting network it is imperative that the relay node must be placed at locations where they can harvest maximum amount of energy. Such locations are candidate locations where the energy harvesting potential known a priori. The goal is also to place a minimum number of relay nodes, to gain connectivity or survivability for the continuous and prolonged network operation.

Alireza Seyedi et.al. in 2008 proposed a unified model based on Markov chain for energy harvesting nodes in [5]. It provided an approximation of probability of event of loss of energy. It also provided an analytical vulnerability metric named as average time to energy run-out to measure the performance of the network.

In 2004, O.Younis and S.Fahmy proposed HEED protocol ( Hybrid Energy Efficient Distributed clustering Protocol ) [6]. Based on the concepts of LEACH , it used residual energy as primary metric to select cluster. It used other network features such as communication cost and node degree as other parameter to break ties among different sensor nodes that qualify to be cluster heads. The scheme works in a number of iterations to perform clustering. The probability of becoming 
cluster head doubles for a node which is not selected by any Cluster head.

In 2014, P.Priya presented ASH-HEED protocol for Heterogeneous WSNs [7]. This protocol assigned the nodes with different levels of heterogeneity based on energy for increasing the network lifetime. A node scheduling scheme classifies nodes into Active and Sleep nodes. By introducing 2-level heterogeneity the sensor nodes are named as Advanced and Normal nodes. $30 \%$ more advanced nodes are deployed that have $150 \%$ more energy than the normal nodes. In case of 3-level heterogeneity the sensor nodes are named as Super, Advanced and Normal nodes. The super nodes are deployed $20 \%$ more in numbers and have $150-300 \%$ more energy than the normal nodes.

Bongsue Suh et.al. in 2009 provided a strategy for data forwarding optimally by minimizing sleep duration time wireless sensor networks that has energy harvesting sensor nodes. Based on the values of remaining energy and harvested energy of sensor nodes, the base station (data sink) determines the sleep duration time and the data forwarding strategy for the next data gathering round [11]. All sensor nodes act as a relay node. They receive and forward the data to the data sink. This guarantees that not all sensor nodes reach the energy low. Minimizing sleep duration time, results in the maximum data gathering

\section{SYSTEM MODEL}

In this paper the aim is to gain maximum output from the network in terms of longer network lifetime as these networks are usually designed and used for strategic information gathering in the hostile areas. For this one needs to provide the continuous supply of power or energy to the system so that the system can always providing the services. The system should survive and stay connected for a prolonged network lifetime. The approach to achieve this goal can be either constraint the use of energy and save power or generate the energy from the ambient sources i.e. energy harvesting.

\subsection{Network Architecture and Framework}

The network is heterogeneous in nature. The model consists of a sink, static sensor nodes (SNs) and Actuator nodes(ANs) The sink is the intermediate between the sensor network and the end user or we can say it is a gateway between the sensor network and the outside world. The sink collects data from the sensor network and forwards it to the end user. The sink can also send data or command to the sensor nodes. It monitors the network continuously and is responsible for implementing policies for strategic operation.

It is assumed that the sink is connected to an unlimited energy source and has no energy constraint. The sensor nodes (SNs) are static in nature and all have similar transmission range. Since they all are battery powered, therefore, after operating for certain time they will be discharged. The Actuator nodes (ANs) are capable of energy harvesting. They act as cluster heads and relay nodes to communicate the sensed data from the sensor nodes to the sink node. Since they have to communicate a large number of packets therefore they tend to be dead soon by loosing energy. Therefore they are equipped with energy harvesting capability. Their density in the network is low.

\subsection{Network Deployment and Initialization}

The network is deployed randomly as shown in the figure 1 . There is only one base station in the network, whereas, the sensor nodes have high density in the network. A small density of energy harvesting Actuator nodes is also deployed.
They are tend to have say 5\% of total nodes whereas the sensor nodes tend to be $95 \%$ of the total nodes. If total $\mathrm{N}$ number of nodes are deployed in the network then $\mathrm{N}=\mathrm{Ns}+\mathrm{Na}$

(1)

where Ns is the number of sensor nodes and $\mathrm{Na}$ is number of actuator nodes.

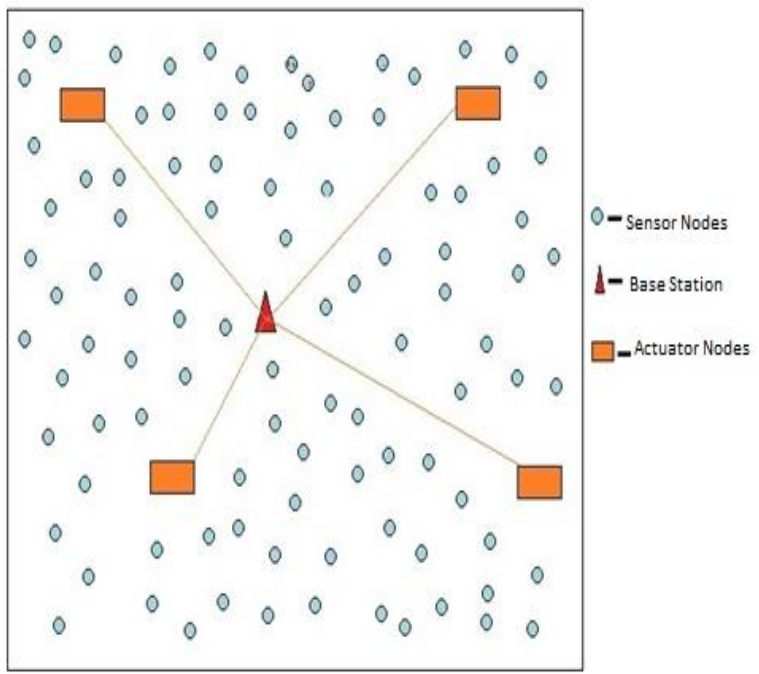

Fig 1: Deployment of nodes in the network

The ANs send a solicitation message to the sensor nodes in its transmission range to form a group. All the sensors that receive this message reply back to the $\mathrm{AN}$ to join its group. If a SN gets message from multiple ANs then it can join the AN with high residual energy. The ANs report their degree of nodes connected to them to the sink node. If the total number of member nodes of all the ANs is not equal to the total number of nodes deployed in the network then the sink can command the ANs to increase their transmission range and reconfigure the network, depending upon the policy being used.

\subsection{Data Reporting Model}

The SNs sense the data and send to AN, which in turn sends the data to the sink. In a similar way sink sends the command to the AN, which then forwards it to the SNs. Each AN maintains a Set $\mathrm{i}\left(\mathrm{AN}_{\mathrm{i}}\right)$ which is a set of nodes in the radio range of $\mathrm{AN}_{\mathrm{i}}$. Initially sink send a command for network formation. The ANs receive the command and forward to other ANs and a solicitation message to sensor nodes. The ANs that do not receive message/ command from the sink directly forms a multi-hop path through other ANs for communication to the sink. The other SNs follow the singlehop path for communication with the sink.

\subsection{Energy Dissipation Model}

For each $\mathrm{SN}_{\mathrm{i}}$, Energy dissipated to transmit a B bit packet over a distance $\mathrm{S}$ to $\mathrm{AN}_{\mathrm{i}}$ of its group is

$\mathrm{E}_{\operatorname{Trs}_{\mathrm{i}}}(\mathrm{B}, \mathrm{S})=$ B. $\mathrm{E}_{\mathrm{b}}+$ B. $\epsilon_{\mathrm{fs}_{\mathrm{s}}} * \mathrm{~S}_{\text {toAN }_{\mathrm{i}}}^{2}$

Energy dissipated in transmitting a total of no of packets $\mathrm{N}_{\text {ptcd }_{i}}$ and receiving a total no. Of packets $\mathrm{N}_{\mathrm{rcd}_{i}}$ is

$\mathrm{E}_{\mathrm{D}_{\mathrm{i}}}=\mathrm{N}_{\mathrm{ptcd}_{\mathrm{i}}} * \mathrm{E}_{\operatorname{Trs}_{\mathrm{i}}}+\mathrm{N}_{\mathrm{rcd}_{\mathrm{i}}} * \mathrm{E}_{\mathrm{rec}_{\mathrm{i}}}$

Where energy $E_{\text {rec }_{i}}$ is the energy dissipated in receiving a message of bits $\mathrm{B}$ is

$\mathrm{E}_{\mathrm{rec}_{\mathrm{i}}}=\mathrm{B} \cdot \mathrm{E}_{\mathrm{b}}$ 
Therefore energy remaining for any $\mathrm{SN}_{\mathrm{i}}$ at any instant of time is will be

$E_{R_{i}}=E_{T_{i}}-E_{D_{i}}$

Whereas the energy remaining for any $\mathrm{AN}_{\mathrm{i}}$ at any instant of time is will be

$\mathrm{E}_{\mathrm{R}_{\mathrm{i}}}=\mathrm{E}_{\mathrm{T}_{\mathrm{i}}}-\mathrm{E}_{\mathrm{D}_{\mathrm{i}}}+\mathrm{E}_{\mathrm{H}_{\mathrm{i}}}$

Where $E_{H_{i}}$ is the energy harvested by any $\mathrm{AN}_{\mathrm{i}}$.

The cumulative harvested energy by all ANs in round $\mathrm{j}$ is

$\mathrm{E}_{\mathrm{C}_{\mathrm{j}}}=\sum \mathrm{E}_{\mathrm{H}_{\mathrm{i}}}$

Cumulative Residual Energy of the network at any time is

$\mathrm{E}_{\mathrm{N}}=\mathrm{E}_{\mathrm{N}_{\mathrm{a}}}+\mathrm{E}_{\mathrm{N}_{\mathrm{S}}}$

The probability index for harvesting energy predicts the probability of harvesting in the next rounds depending on the energy harvested in previous round. It can be calculated as per the equation

$P_{j}=P_{j-1} * E_{C_{j}-1} / E_{C_{j-1}}$

Where $P_{j-1}$ is probability index for round $j-1$.

This probability index thus provides an approximation for the energy to be remained and to be collected in the subsequent round.

\subsection{Policy Management and Shift}

The base station or sink controls the strategic operation of the network. It initiates the network formation under either of the twin policies of energy management. The network initiates with the policy 1 .

Policy1: the network is assumed to be initialized with this policy. The data reporting model in this policy will be continuous. All the sensor nodes stay in alert mode. The SNs sense and then report data continuously to the ANs which send data to the sink.

The probability index for the future rounds has value more than a threshold value. If the value goes below the threshold value then the sink immediately switches the network into second mode by broadcasting the message in the network.

Policy2: In this mode the network turns into an event triggered mode. The sensor nodes only communicate data if an event occurs. Thus this policy decreases the load of transmitting and receiving packets.

If the ANs in this mode acquire enough energy then the Cumulative residual energy of the network is likely to rise and therefore the network can shift back to 1st mode. If the residual energy do not improve then the probability index will keep on decreasing and thus the network will continue operating in the second policy mode only.

\section{PERFORMANCE EVALUATION}

Table 1 provides an insight of the initial values of various metrics and setup of the system. It includes total number of sensor nodes, Actuator nodes, size of the network, channel bandwidth etc.
Table 1: Characteristics of the Test Network

\begin{tabular}{|l|l|}
\hline Parameter & Value \\
\hline Number of Sensor Nodes & 100 \\
\hline Number of MPN & 04 \\
\hline Network Size & $100 \mathrm{~m}^{*} 100 \mathrm{~m}$ \\
\hline Base Station Location & $(50,50)$ \\
\hline Channel Bandwidth & $1 \mathrm{Mb} / \mathrm{s}$ \\
\hline Radio Propagation Speed & $3 * 103 \mathrm{~m} / \mathrm{s}$ \\
\hline Initial Energy of MPN & 50 Joules \\
\hline Initial Energy of each node & .005 Joule \\
\hline
\end{tabular}

Table 2 provides the network experimental figure of energy consumed in transmitting and receiving one bit of data. Therefore the energy consumed to transmit or receive a packet of 500 bytes can easily be calculated.

Table 2: Energy Related Parameters

\begin{tabular}{|l|l|}
\hline \multicolumn{1}{|c|}{ Parameter } & \multicolumn{1}{c|}{ Value } \\
\hline ETX & $50 * 0.000000001 \mathrm{nj} / \mathrm{bit}$ \\
\hline ERX & $50 * 0.000000001 \mathrm{nj} / \mathrm{bit}$ \\
\hline EDA & $5 * 0.000000001$ \\
\hline Pktsize & 500 bytes \\
\hline
\end{tabular}

The initial deployment of the sensor network can be observed from the figure 2. The sink or Base station is centered in the network field and remains fixed in nature. The network is randomly deployed. Also the $4 \mathrm{ANs}$ are deployed in network field. The sensor node will sense and transmit the data to ANs, ANs will send the data to the sink. The sink will forward it to the end user.

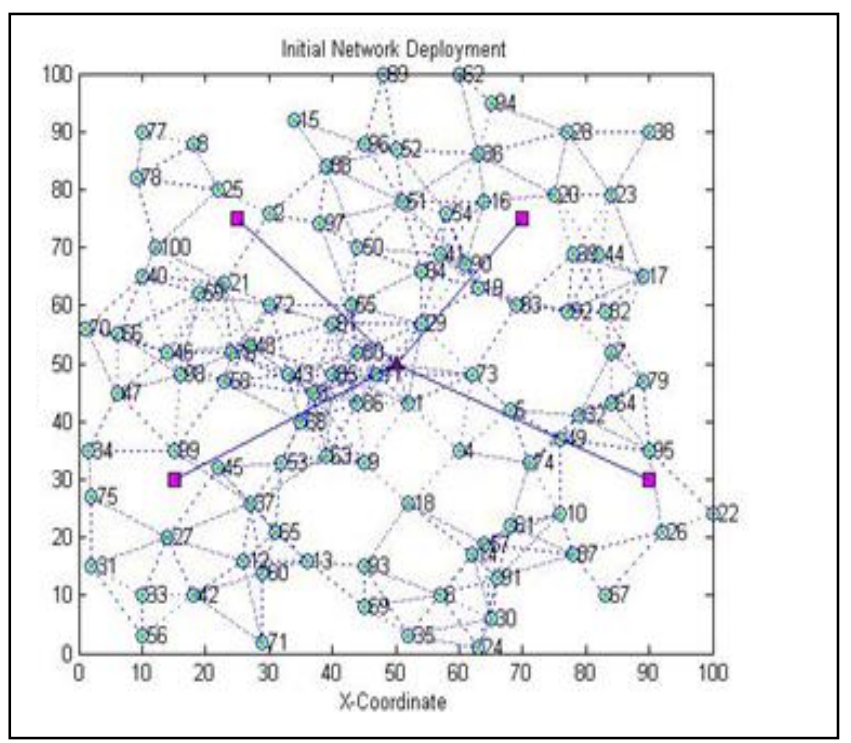

Fig 2: Initial Network Deployment

Figure 3 shows that the number of nodes are dead more in case of absence of policy 2. Whereas, if twin policies are available then the number of dead nodes are less in every round of network operation. The $\mathrm{X}$-axis represents the number of rounds .The Y-axis represents the number of dead nodes. 


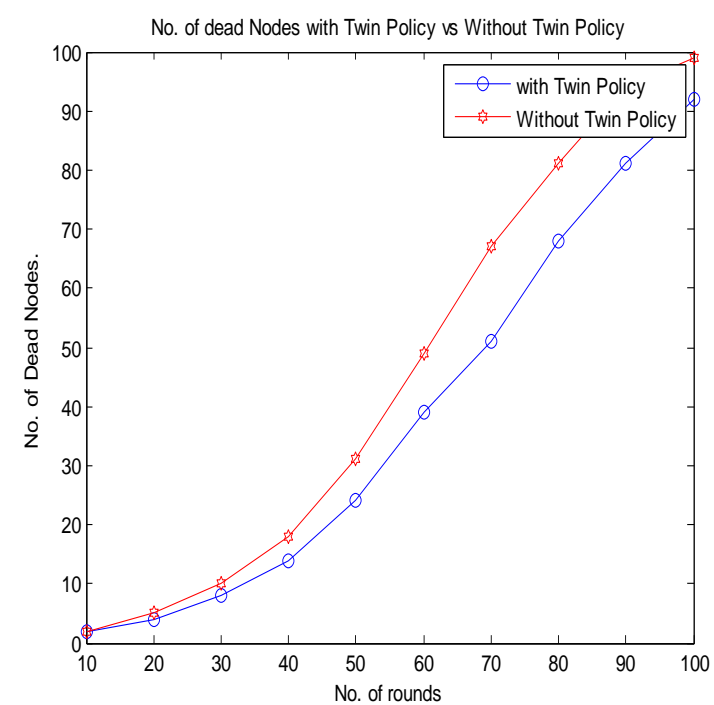

Fig 3: Number of dead nodes per round with twin policy vs without twin policy.

\section{CONCLUSION AND FUTURE SCOPE}

This model considers the network to work in either continuous mode or in the event triggered mode. It saves lots of energy by restricting to second policy and thus can keep on providing strategic data in the hostile area. In future a new policy can be introduced for the network to switch in query driven mode as well which can not only save energy but also can increase the application area of this scheme. One can also provide a switch between query driven and event driven policies which can certainly provide the longer network lifetime.

\section{REFERENCES}

[1] Winston K.G. Seah, Zhi Ang Euy, Hwee Pink Tan, "Wireless Sensor Networks Powered by Ambient Energy Harvesting (WSN-HEAP) - Survey and Challenges", in proceedings IEEE ICC 2010.

[2] T. Voigt, H. Ritter, and J. Schiller. "Utilizing Solar Power in Wireless Sensor Networks", In Proc. of the 28th Annual IEEE Intl. Conf. on Local Computer Networks (LCN '03), Bonn/Konigswinter, Germany, Oct. 2003.

[3] J. Jeong, X. F. Jiang, and D. E. Culler. "Design and Analysis of Micro-Solar Power Systems for Wireless
Sensor Networks", In Proc. of the 5th Intl. Conf. on Networked Sensing Systems (INSS '08), Kanazawa, Japan, June 2008.

[4] Satyajayant Misra, Nahid Ebrahimi Majd, Hong Huang, "Constrained Relay Node Placement in Energy Harvesting Wireless Sensor Networks", Eighth IEEE International Conference on Mobile Ad-Hoc and Sensor Systems, 2011.

[5] Alireza Seyedi, Biplab Sikdar, "Modeling and Analysis of Energy Harvesting Nodes in Wireless Sensor Networks", Forty-Sixth Annual Allerton Conference Allerton House, UIUC, Illinois, USA September 23-26, 2008.

[6] OssamaYounis and Sonia Fahmy, "Distributed Clustering in Ad-hoc Sensor Networks: A Hybrid, Energy-Efficient Approach", in Proceedings of IEEE INFOCOM, Hong Kong, March 2004, an extended version appeared in IEEE Transactions on Mobile Computing, 3(4), Oct-Dec 2004.

[7] P.Priya, Rupeshsah, P.saravanan, Dr.J.Senthil Kumar, "ASH-HEED Protocol for Heterogeneous Wireless Sensor Networks", in International Journal of Innovative Research in Computer and Communication Engineering, pp. 60-66, Vol.2, Special Issue 5, October 2014.

[8] D. Niyato, E. Hossain, Afshin Fallahi, "Sleep and wakeup strategies in solar powered wireless sensor/mesh networks: performance analysis and optimization", IEE Trans. On Wireless comm., vol. 6, no. 2, pp. 221-236, Feb 2007.

[9] Michael R. Hansen, Mikkel Koefoed Jakobsen and Jan Madsen, "A Modeling Framework for Energy Harvesting Aware Wireless Sensor Networks" in Sustainable Energy Harvesting Technologies - Past, Present and Future, 2011, pp. 3-24.

[10] W. Michael, T. D. Klaus, H. Kester, B. Graeme, "Theoretical and practical aspects of military wireless sensor networks", Journal of Telecommunications and Information Technology, 2008, pp. 37-45.

[11] Bongsue Suh, Chulho Won, Seong-Woo Kim," Minimizing sleep duration time for energy harvesting wireless sensor networks", IEEE SENSORS 2009 Conference. 